\title{
WAVELET ANALYSIS OF HUMAN PHOTORECEPTORAL RESPONSE
}

\author{
R. Barraco, D. Persano Adorno, and M. Brai \\ Dipartimento di Fisica e Tecnologie Relative, Università di Palermo, Palermo, Italy
}

\begin{abstract}
Feature detection of biomedical signals is crucial for deepening our knowledge of the physiological phenomena giving rise to them. To achieve this aim, even if many analytic approaches have been suggested only few are able to deal with signals whose features are time dependent, and to provide useful clinical information. In this work we use the wavelet analysis to extract peculiarities of the early response of the photoreceptoral human system, known as $a$-wave ERG-component. The analysis of the $a$-wave features is important since this component reflects the functional integrity of the two populations of photoreceptors, rods and cones whose activation dynamics are not well known. Moreover, in incipient photoreceptoral pathologies the eventual anomalies in $a$-wave are not always detectable with a naked eye analysis of the traces. We here propose the possibility to discriminate the pathologic from the healthy traces throughout the differentiation of their time-frequency characteristics, revealed by the wavelet analysis. The investigated pathologies are the Achromatopsia, a cone disease and the Congenital Stationary Night Blindness, a rod trouble. The results show that the number of stable frequencies present and their times of occurrence are indicative of the status of the retinal photoreceptors. In particular, in the pathological cases, the frequency components shift toward lower values and change their times of occurrence, with respect to healthy traces.
\end{abstract}

Keywords-component; wavelet analysis, photoreceptoral response, Achromatopsia, Congenital Stationary Night Blindness.

\section{INTRODUCTION}

One of the main goals in biomedical data processing is to extract clinically relevant information in terms of parameters characterizing the biosignal to improve medical diagnosis. The investigation of biomedical signals is of fundamental importance both in the study of the functionality of the systems that generate them and in the early diagnosis of eventual diseases. The analysis of biomedical signals, because of their non stationary behaviour, requires the use of analysis techniques complementary to those generally applied (FFT, SFT etc.), capable of giving also the temporal occurrence of the frequency components. In recent decades wavelet analysis (WA) is beginning to play a central role in many biomedical applications thanks to its ability to represent the signal in a time-frequency domain and to analyse non-linear and nonstationary responses. Its applications to various biomedical signals such as ECG, EEG, EMG [1-3] are widespread and different. This study proposes the WA as methodological approach to the investigation and evaluation of the status of the working photoreceptors in the human retina. This purpose has been accomplished through the analysis of a component ( $a$-wave) of the electroretinogram (ERG), that characterises the retinal response to a flash stimulation [4-5]. In fact, the $a$ wave is related to the activities of the photoreceptors, rods and cones, assigned to scotopic (vision in moderate levels of illumination) and photopic vision, respectively. In particular, we investigate a progressive cone disease, the Achromatopsia (ACR), and the Congenital Stationary Night Blindness (CSNB), a non-progressive rod trouble.

\section{Materials ANd Methods}

\section{A. Wavelet Analysis}

Time-frequency analysis is crucial in the extraction of features characterizing biosignals. Traditional Fourier transform provides only information about the spectral content of a signal, thus it is not suitable for the analysis of non-stationary signals [6]. Continuous Wavelet Transform (CWT) overcomes these problems using variable-length windows (small windows capture the high frequency content providing good temporal localization; large windows capture the low frequency content ensuring good frequency resolution). The kernel functions used in wavelet transform [6-7] are derived from a prototype function (mother wavelet) by operation scaling and / or translation controlled by the scale parameter $\sigma$ (related to the frequency band) and the translation parameter $\tau$ (related to the temporal position of the window). High values of $\sigma$ allow rough analysis of the signal. Conversely, low values for $\sigma$ can "capture" the detail information. The coefficients of CWT can be easily calculated using the equation:

$$
W T(\tau, \sigma)=\frac{1}{\sqrt{\sigma}} \int_{-\infty}^{+\infty} x(t) \psi^{*}\left(\frac{t-\tau}{\sigma}\right) d t
$$

where $x(t)$ is the signal to be analysed and $\psi$ is the chosen wavelet. The choice of the wavelet depends on the type of the signal to be processed and on the information to be acquired. We have chosen the Mexican Hat (MhW, second derivative of a Gaussian) that is continuous, non orthogonal, suitable to evidence the behaviour of the signal in regions of stationary activity It is defined by: 


$$
M H W(t, \tau, \sigma)=\frac{1}{\sqrt{2 \pi}}\left[1-\left(\frac{t-\tau}{\sigma}\right)^{2}\right] \cdot e^{-\frac{1}{2}\left(\frac{t-\tau}{\sigma}\right)^{2}}
$$

As a consequence of the Heisenberg uncertainty relation, the WA gives good time resolution and poor frequency resolution at small scales (high frequencies), whereas it provides good frequency resolution and poor time resolution at large scales (low frequencies). The $a$-waves were processed using the toolbox of MatLab. We, here, have chosen to display the results in terms of $\mathrm{WT}_{\mathrm{abs}}(\sigma, \tau)$ defined by:

$$
W T_{a b s}(\sigma, \tau)=\frac{a b s(W T(\sigma, \tau))}{\max [a b s(W T(\sigma, \tau))]}
$$

Time-frequency spectrum is then shown as a shaded contour plot of $\mathrm{WT}_{\mathrm{abs}}(\sigma, \tau)$. The correlation magnitudes are expressed as a pink-scale of a scalogram. The WA shows the correlation existing with the $\mathrm{MH}$ through the existence of clusters in the corresponding scalogram [8-9].

\section{B. Main features of the ERG}

The electroretinogram (ERG) is the electrical response of the retina to a flash stimulation [10-11]. It is a sequence of components generated in the different retinal layers. The first component, known as $a$-wave is, in normal subjects, a negative potential marked by two dips, denoted as $a_{1}$ and $a_{2}$, whose origin is generally attributed to the contribution of cone and rod activities, respectively. The $a$-wave is followed by the positive $b$-wave, arising from the activation of deeper retinal layers and by other waves.

A photoreceptoral pathology can slightly affect the $a$-wave in manner not always naked eye easily detectable or substantially modify it. The pathologies chosen here, ACR and CSNB represent two different examples of these possibilities. Fig. 1 reports the ERGs belonging to these groups of subjects recorded under the same experimental conditions. Panel (a) shows 10 ERGs of healthy subjects; panels (b) and (c) display 8 ACR and 6 CSNB patients, respectively. ACR is a hereditary disease due to a lack of cone vision. There are two forms in which ACR can subsist: incomplete and complete. In particular, we have analysed the behaviour of the $a$-waves belonging to the patients affect by incomplete ACR. In general, the eyes affected by ACR, lacking normal cone vision, are not able to adapt normally to higher levels of illumination; the ACR patients are completely colour-blind, or nearly so, and have very poor visual acuity. Because there are many variations in the severity of the pathology among individual achromats, it is not simple to identify its different stages.

CSNB is a rare inherited non-progressive disorder of the retina involving rods. CSNB patients have a reduced sharpness of vision, night blindness and have difficulty in adapting to low light situations due to impaired photoreceptor transmission.

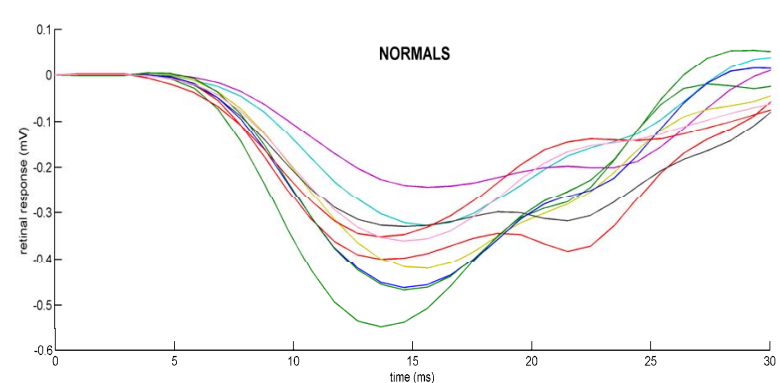

(a)

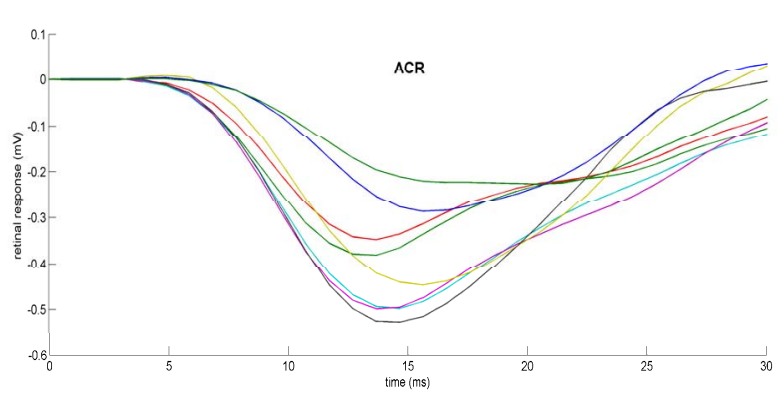

(b)

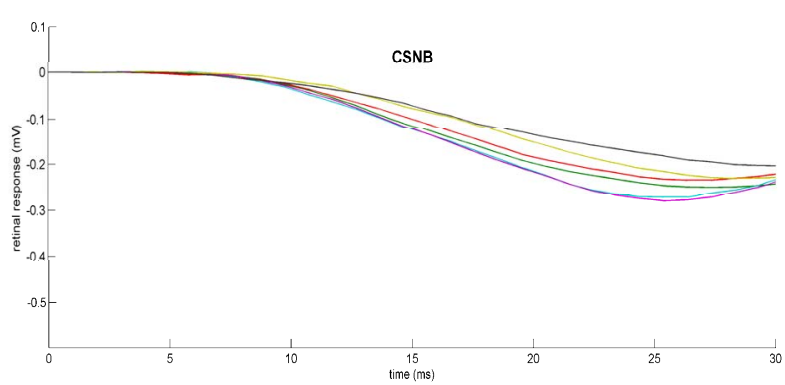

(c)

Figure 1. ERG samples (truncated at $30 \mathrm{~ms}$ ) recorded with luminance value $\log (\mathrm{I} / \mathrm{I} 0)=1.0$. The sample of human healthy ERGs, the ACR sample and CSNB sample are shown in (a), (b) and (c) panel, respectively.

\section{ERG acquisition}

The ERGs were recorded following routine methods by means of Henke's corneal electrodes [12-13]. The electrodes were frontal, with the ground electrode on the forehead. The signal was suitably filtered and amplified in line with the ISCEV standards [12]. The standard luminance was $1.7 \mathrm{~cd} \mathrm{~s}$ $\mathrm{m}^{-2}$ and the duration of the flash $50 \mu \mathrm{s}$. All patients were subjected to maximal papillary dilatation $(\geq 7 \mathrm{~mm})$ with Nethyl( $\alpha$-picolyl) tropicamide $1 \%$, and the cornea was anesthetized using oxybuprocaine hydrochloride 4\%. They were then darkadapted (scotopic ERG) for $30 \mathrm{~min}$, in accordance with the standards for clinical electroretinography [12-13]. Each exam lasted $500 \mathrm{~ms}$ with sampling time $\delta \mathrm{t}=0.1953 \mathrm{~ms}$. Each trace was obtained by averaging at least 3 responses. Data were sampled with a Nyquist frequency of $2560 \mathrm{~Hz}$ and stored as ASCII files for subsequent retrieval and analysis. The time interval used in the figures spans over $30 \mathrm{~ms}$. At greater times, an overlap between the tail of the $a$-wave and the beginning of the successive $b$-wave begins to take place.

\section{RESULTS AND DISCUSSION}

The present wavelet analysis provides a time-frequency representation of the $a$-wave through the individuation of a set 


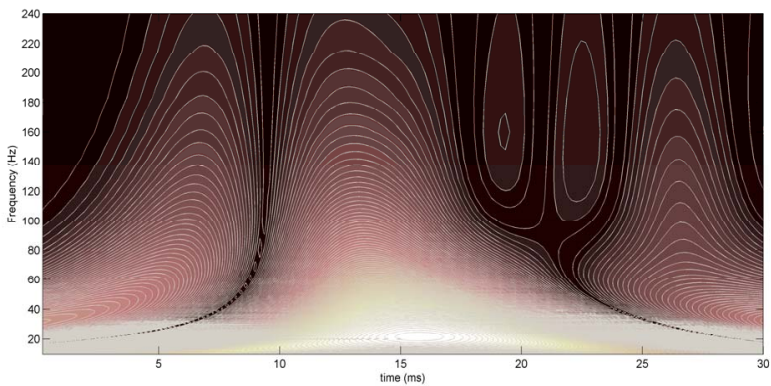

(a)

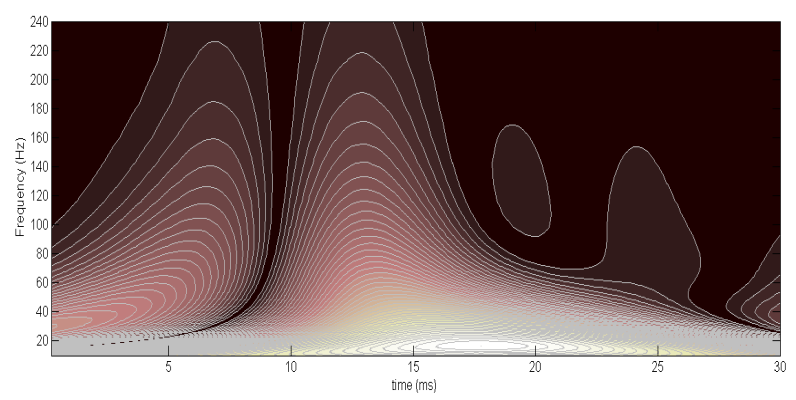

(b)

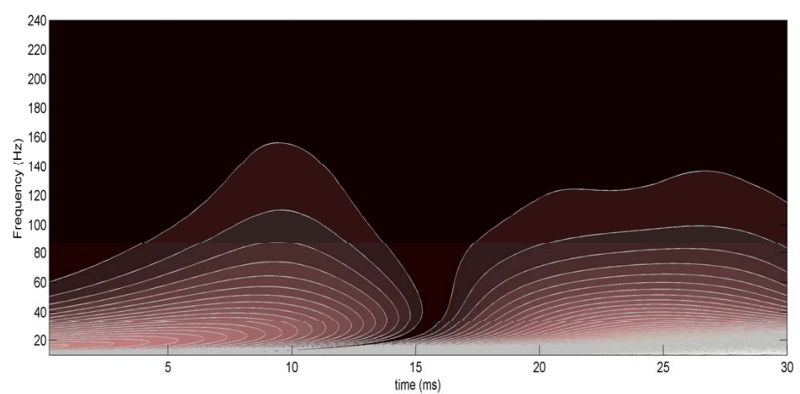

(c)

Figure 2: Spectral behaviour of the ERGs shown in Fig. 1. The panels (a), (b) and (c) show the amplitude of the transform in the range from 10 to $240 \mathrm{~Hz}$ across time as a colour scalogram, for a representative subject of the healthy sample, ACR and CSNB sample, respectively. A scale of colours tending to white denotes the high-energy components, the pink scale denotes the middle energy the low-energy components in the $30 \mathrm{~ms}$ time interval. The MatLab package has been used.

of frequencies characterizing the traces. The relevant frequencies are identified through the existence of clusters in the colour scalograms (Fig.2) that show the correlations among $\mathrm{MH}$ and signal. Each scalogram yields a dominant maximum (white cluster) $f_{0}$ and one or two local maxima, $f_{l}$ and $f_{2}$. Panels of Fig. 2 show the spectral behaviour of a representative normal subject and of an ACR and a CSNB patient, recorded under the same luminance conditions as in Figure 1. The three panels report the amplitudes of the wavelet transform in the $10-240 \mathrm{~Hz}$ range across time as colour contour plots, in which the correlation magnitudes are expressed in a pink scale. To optimize the characterisation of the $a$-waves, taking into account behaviour differences concerning the consistency of the dynamics across healthy and pathological subjects, both the mean values of the frequencies $f_{0}, f_{1}$ and $f_{2}$ and their times of occurrence were analysed. Table 1 furnishes the most relevant data about the statistical parameters regarding normal, $\mathrm{ACR}$ and $\mathrm{CSNB}$ traces. It reports the frequency and time mean values, their standard deviations (SD), the appearances (in how many subjects a certain frequency group can be identified) of the stable
TABLE I. VALUES OF THE FREQUENCY COMPONENTS, TIMES OF OCCURRENCE, APPEARANCES EXTRACTED FROM THE THREE SAMPLES OF TRACES FOR LUMINANCE VALUE LOG $\left(\mathrm{I} / \mathrm{I}_{0}\right)=1.0$.

\begin{tabular}{|c|c|c|c|}
\hline Sample & Freq. $(\mathbf{H z}) \pm \boldsymbol{S D}$ & Time $(\mathrm{ms}) \pm \boldsymbol{S D}$ & Appearance (\%) \\
\hline \multirow{4}{*}{ Norm. } & $f_{0}: 19.9 \pm 1.5$ & $t_{0}: 17.2 \pm 0.8$ & 100 \\
\cline { 2 - 4 } & $f_{1}: 139.4 \pm 6.4$ & $t_{1}: 25.3 \pm 2.7$ & 50 \\
\cline { 2 - 4 } & $f_{2}: 171.4 \pm 12.2$ & $t_{2}: 20.6 \pm 2.2$ & 80 \\
\hline \multirow{4}{*}{ ACR } & $f_{0}: 18.3 \pm 2.9$ & $t_{0}: 17.5 \pm 0.9$ & 100 \\
\cline { 2 - 4 } & $f_{1}: 111.2 \pm 11.3$ & $t_{1}: 21.7 \pm 2.7$ & 62.5 \\
\cline { 2 - 4 } & $f_{2}:------$ & $t_{2}:------$ & ----- \\
\hline \multirow{3}{*}{ CSNB } & $f_{0}: 10.2 \pm 0.20$ & $t_{0}: 31.25 \pm 1.1$ & 100 \\
\cline { 2 - 4 } & $f_{1}:------$ & $t_{1}:------$ & ----- \\
\cline { 2 - 4 } & $f_{2}:------$ & $t_{2}:------$ & ------ \\
\hline
\end{tabular}

frequency components. A comparison of the data in the table, indicates that the sets of normal and ACR subjects reveal minor changes in $f_{0}$ and significant differences in $f_{1}$ and $f_{2}$, whereas the CSNB patients present a appreciable difference in $f_{0}$. In particular, two effects are evident in the pathological cases: i) a general reduction of the average frequency values; ii) the absence of $f_{2}$ in the ACR sample and of $f_{1}$ and $f_{2}$ in CSNB. These outcomes provide relevant information about the behaviour and efficiency of the photoreceptors in the three samples. The lower frequency $f_{0}$ is associate with the summed activities of the photoreceptors, in the sense that its value takes into account the temporal distribution of the amount of excited photoreceptors [9]. An alteration of the response in the ACR $a$-waves indicates a reduced contribution of working cones and the deficit of the rod contribution in patients with CSNB. The higher frequencies $f_{1}$ and $f_{2}$ are related to the contributions of the photoreceptors ( $f_{1}$ to rods, $f_{2}$ to cones) and to their peculiarities. Their presence is indicative of the functional integrity of the retina. In fact, in ACR traces, where the rod population correctly works, $f_{l}$ is present. On the other hand, a rod pathology, represented by the CSNB $a$-waves, leads to the lacking of both frequency components. In CSNB, $f_{l}$ is absent because it is related to rod activity whereas $f_{2}$, bound to cones, does not appear since is necessary a higher luminance value for switching it.

\section{CONCLUSION}

The wavelet analysis, carried out over healthy and pathological subjects, has allowed us to differentiate the traces through the identification of peculiar time frequency features of the $a$-wave. Moreover, since both pathologies only affect one of the two photoreceptoral families, it was possible to confirm our associations about the three stable frequency components and the related photoreceptoral activities. Our findings indicate that the eventual absence of one or two frequency components and/or its shift toward lower values and different times of occurrence can be related to the pathological response of one photoreceptoral population. In fact, the lowering of the frequency values is linked with the improper activity of the cones or the rods that affects the crosstalk within the same photoreceptoral population or between rods 
and cones. This circumstance alters the normal set up of correlation phenomena that characterize the healthy response of the photoreceptors. This assessment validates the current knowledge about rod-cone interactions during the response in normal subjects and provides useful information about the frequency-temporal features of the two pathologies and their clinical interpretation. Although the analytical approach provided by the WA needs a further update, both in terms of methodology and increasing of the statistical sample, it could be useful for the recognition task and classification of the $a$ wave into different patho-physiological disease categories.

\section{REFERENCES}

[1] S. Pal and M. Mitra, "Detection of ECG characteristic points using Multiresolution Wavelet Analysis based Selective Coefficient Method, Measurement “43, 2010, pp. 255-261.

[2] C. Saritha, V. Sukanya, Y. Narasimha Murthy, "ECG Signal Analysis Using Wavelet Transforms", Bulg. J. Phys. 35, 2008, pp. 68-77.

[3] X. Hu, Q. Yu, W. Liu, J. Qin, "Feature Extraction of Surface EMG Signal Based on Wavelet Coefficient Entropy", IEEE Xplore, 978-14244-1748-3/08.

[4] T. D Lamb, E. N. Pugh, A quantitative account of the activation steps involved in phototransduction in amphibian photoreceptors, Journal of Physiology 449, 1992, pp. 719-758.
[5] Cideciyan A.V., Jacobson S.G. "An alternative phototransduction model for human rod and cone ERG a-waves: normal parameters and variation with age", Vision Res 36, 1996, pp. 2609-21

[6] O. Rioul, M. Vetterli, "Wavelet and signal processing" IEEE SP Magazine, 1991, pp. 14-38

[7] N. M. Astaf'eva, "Wavelet analysis: basic theory and some applications", Physics-Uspekhi Fizicheskikih Nauk 39, 1996, pp. 10851108.

[8] S. Varadharajan, K. Fitzgerald, V. Lakshminarayanan, "A novel method for separating the components of the clinical electroretinogram", J Mod Opt 54, 2007, pp. 1263-1280.

[9] R. Barraco, D. Persano Adorno and M. Brai, "ERG Signal Analysis Using Wavelet Transform" accepted for the pubblication in Theory in Biosciences.

[10] M. E. Burns, T. D. Lamb, "Visual Transduction by Rod and Cone Photoreceptors in Chalupa", in The Visual Neurosciences, eds. L M and Werner J S pp. 215-33, 2003.

[11] J. G. Robson, S. M. Saszik, J. Ahmed, L. Frishman, "Rod and cone contributions to the a-wave of the electroretinogram of the macaque", Journal of Physiology 547. 2, 2003, pp. 509-530.

[12] Marmor et al. "International Standardisation Committee, Standard for clinical electroretinography", Arch ophthalmol 107 (1989) 816-819

[13] F. Marmor, G. E. Holder, M. W. Seeliger, S. Yamamoto, Standard for clinical electroretinography (2004 update) Doc. Ophthalmol 108 (2004) 107-114. 